\title{
La dirección estratégica en la universidad pública: una investigación en las universidades tecnológicas de México
}

Strategic Management in Public Universities: A Research in Mexican Technological Universities A direção estratégica na universidade pública: uma pesquisa nas universidades tecnológicas do México

\author{
Eugenio César Velázquez Santana* \\ Miguel Cruz Vásquez ${ }^{* *}$ \\ José Alfredo López de Cosío ${ }^{* \star}$
}

Fecha de recibido: 25 de febrero de 2014

Fecha de aprobado: 17 de septiembre de 2014

Doi: dx.doi.org/10.12804/rev.univ.empresa.28.2015.04

* Doctorado en Planeación Estratégica y Dirección de Tecnología de la Universidad Popular Autónoma del Estado de Puebla (México), Maestría en Tecnologías de la Información de el Instituto Tecnológico de Zacatepec (México), Ingeniero en Sistemas Computacionales del Instituto Tecnológico de Zacatepec (México). Profesor Investigador de la División Académica de Tecnologías de la Información de la Universidad Tecnológica Emiliano Zapata del Estado de Morelos (México). Correo electrónico: cesarvelazquez@utez.edu.mx. Dirección postal: Avenida Universidad Tecnológica No. 1, Colonia Palo Escrito, Código Postal 62760, Emiliano Zapata, Morelos, México.

** Doctorado en Economía de la Universidad de las Américas Puebla UDLAP (México), Maestría en Políticas Públicas del Instituto Tecnológico Autónomo de México ITAM (México), Ingeniero Industrial Eléctrico del Instituto Tecnológico de Oaxaca (México). Profesor Investigador del Posgrado en Planeación Estratégica y Dirección de Tecnología de la Universidad Popular Autónoma del Estado de Puebla UPAEP (México). Correo electrónico: miguel.cruz@upaep.mx

*** Doctorado en Planeación Estratégica y Dirección de Tecnología de la Universidad Popular Autónoma del Estado de Puebla (México), Maestría en Administración de la Empresa Industrial de la Universidad Iberoamericana campus Puebla (México), Ingeniero en Sistemas Computacionales del Instituto Tecnológico y de Estudios Superiores de Monterrey campus Monterrey (México). Profesor del Centro de Investigación y Posgrado de la Universidad Popular Autónoma del Estado de Puebla (México).Correo electrónico: josealfredo.lopez@upaep.mx 
Para citar este artículo: Velázquez Santana, E. C., Cruz Vásquez, M., \& López de Cosío, J. A. (2015). La dirección estratégica en la universidad pública: una investigación en las universidades tecnológicas de México. Universidad \& Empresa, 17(28), 87-104. Doi: dx.doi.org/10.12804/rev.univ.empresa.28.2015.04

\section{RESUMEN}

Como sucede también en otro tipo de organizaciones, la dirección estratégica facilita a las Instituciones de Educación Superior (IES) públicas la alineación de su misión, sus recursos y sus capacidades, así como de ellas - como un todo - con su entorno, para lograr el desarrollo de ventajas competitivas. Esta investigación tiene por objetivo estudiar la dirección estratégica de las Universidades Tecnológicas de México. Esto se hace a través de un análisis de la cadena de valor y de las cinco fuerzas competitivas. Este análisis permite identificar algunas mejoras que podrían beneficiar a otras instituciones de este tipo.

Palabras clave: competitividad, dirección estratégica, Instituciones de Educación Superior (IES), universidad pública.

\section{ABSTRACT}

As also happens in another type of organizations, in public Higher Education Institutions (HEI) strategic management facilitates the alignment of their mission, their resources, and their capacities, as well as of themselves - as a single whole - with their environment, in order to create competitive advantages. The purpose of this investigation is to study the strategic management of Mexican Technological Universities through an analysis of the value chain and the five competitive forces. This analysis allows to identify some improvements that could benefit other institutions of this kind.

Keywords: Competitiveness, strategic management, Higher Education Institutions (HEI), public university.

\section{RESUMO}

Como acontece também em outro tipo de organizações, a direção estratégica facilita às Instituições de Educação Superior (IES) públicas o alinhamento de sua missão, seus recursos e suas capacidades, assim como delas - como um todo - com seu entorno, para conseguir o desenvolvimento de vantagens competitivas. Esta pesquisa tem por objetivo estudar a direção estratégica das Universidades Tecnológicas do México. Isto se faz através de uma análise da cadeia de valor e das cinco forças competitivas. Esta análise permite identificar algumas melhoras que poderiam beneficiar a outras instituições deste tipo.

Palavras-chave: competitividade, direção estratégica, Instituições de Educação Superior (IES), universidade pública. 


\section{INTRODUCCIÓN}

Las Instituciones de Educación Superior (IES) públicas enfrentan en la actualidad varios factores que las obligan a plantearse nuevos modelos de gestión. Entre estos se destacan la saturación de la oferta de servicios educativos, los destacados cambios en el sector público y tener que atender a una creciente cantidad de usuarios y servirles de forma distinta, entre otros (Capriotti, 1999). Ante esto, la dirección de la institución universitaria se ve en la necesidad de gestionar adecuadamente sus recursos y capacidades y, en particular, de transformarse, a pesar de que para ella esta no sea una labor sencilla, dados los procesos administrativos que incorpora, los cuales están frecuentemente marcados por una burocracia que no siempre está a favor de los cambios (Bradshaw y Fredette, 2009).

En estos casos, aparece con fuerza la posibilidad de aplicar técnicas y herramientas propias del campo de la dirección estratégica, que es definida por Rodríguez Ponce y Pedraja Rejas (2007), como el proceso por el cual una organización procura alcanzar una ventaja competitiva sustentable que le permita lograr el cambio deseado y tener éxito. Para autores como Olssen y Peters (2005), este tipo de aspectos, así como el cam- bio del modelo interno de gobierno de las universidades, la adopción de técnicas empresariales de gestión, el trabajo en función del alcance de objetivos y el uso de indicadores de actividad, entre otros, responden a la importancia que ha adquirido el conocimiento como un factor esencial para la viabilidad del actual sistema económico de mercado y el destacado rol que ocupan las IES en su difusión, conservación, transformación y creación.

El propósito de este trabajo es identificar la dirección estratégica que siguen las universidades públicas, en particular las Universidades Tecnológicas (UT) de México, con base en el modelo de la cadena de valor, para un análisis interno, y el modelo de las cinco fuerzas, para uno externo. Para esto se ha obtenido información en relación con el estado de la dirección estratégica en universidades públicas de México, con el interés de generar estrategias que les permitan obtener una ventaja competitiva respecto a otras IES.

El artículo está organizado de la siguiente forma: en primera lugar, se presenta el marco teórico, el cual es útil para comprender la educación superior pública tecnológica, la dirección estratégica, los modelos de la cadena de valor y las cinco fuerzas competitivas que define Porter; 
en segundo lugar, se describe la metodología aplicada en esta investigación, así como el desarrollo del análisis interno y externo $\mathrm{y}$, en tercer lugar, se muestran los resultados obtenidos y se concluye haciendo una interpretación de los mismos.

\section{REVISIÓN DE LA LITERATURA}

\subsection{El modelo de la cadena} de valor y su aplicación en la universidad pública

La cadena de valor propuesta por Porter (1985) es una herramienta que permite examinar las actividades que realiza una organización, así como la manera en la que ellas interactúan.
Esto con la finalidad de diagnosticar la creación y mantenimiento de una cierta ventaja competitiva. El análisis de la cadena de valor es una técnica que, en efecto, permite obtener ventajas competitivas para la organización a través de un análisis esencialmente interno (McCormick, 2005).

De acuerdo con Castaño (2004, p. 52), Santos (2005, p. 24) y Gumbau (2006, p. 26), las IES públicas, como otras organizaciones, cuentan también con funciones definidas para el logro de objetivos y, por lo tanto, una herramienta para identificarlas es el uso de la cadena de valor. Esta se presenta, en su versión genérica, en la figura 1 .

Figura 1. Cadena de valor genérica

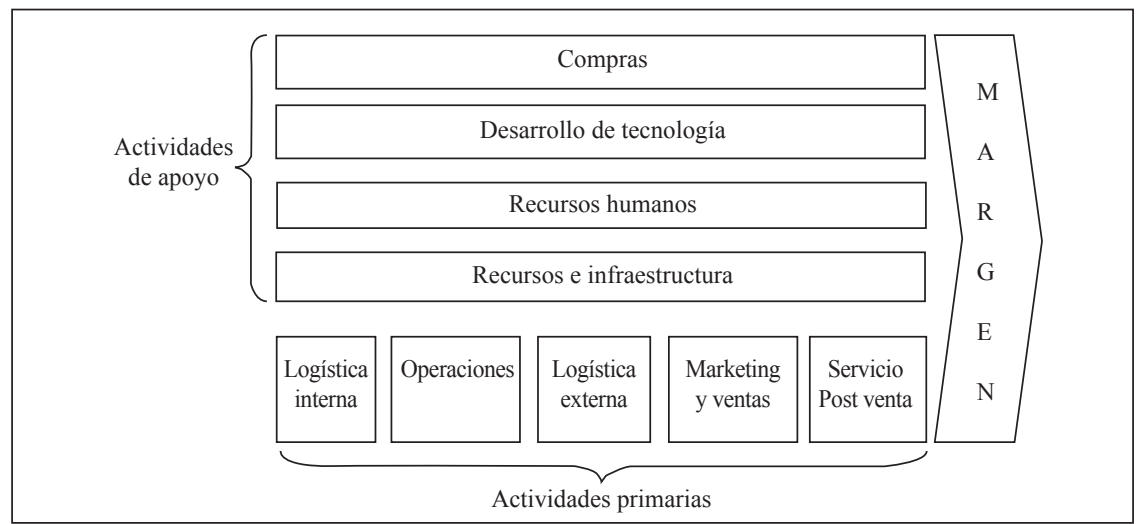

Fuente: traducida y modificada de la figura de Porter (1985, p. 37).

Santos (2005) sugiere que la construcción de la cadena de valor para una universidad se debe realizar en dos pasos: en primer lugar, comparar las actividades de la cadena genérica (ver figura 1) con las de la universidad 
en estudio, esto con el fin de determinar cuáles son las que generan valor para la organización. En segundo lugar, realizar el análisis del medio tanto interno como externo de la organización para determinar las fortalezas, oportunidades, debilidades y amenazas (FODA) de cada una de las actividades de la cadena a desarrollar. Así, por ejemplo, Suárez (2008) utilizó la cadena de valor para realizar un análisis interno, y se apoyó también en el método FODA, con el fin de poder mejorar la productividad académica y la gestión de la Escuela de Postgrado de la Universidad Nacional de Trujillo (Perú). Para hacerlo hizo uso también de otras técnicas de planeación estratégica.

\subsection{El modelo de las cinco} fuerzas y su aplicación en la universidad pública

El modelo de las cinco fuerzas, propuesto por Porter $(1980,1985,2008)$, se basa en que el factor determinante de la rentabilidad de una organización es el nivel de atractivo que tenga el sector. Así, las estrategias competitivas de éxito deberían basarse en la comprensión de los factores que lo determinan. Porter (2008) señala que la rentabilidad de las empresas puede explicarse mediante cinco factores, a saber: rivalidad entre competidores, amenaza de nuevos entrantes, poder de negociación de los proveedores, poder de negociación de los compradores y amenaza de los sustitutos.

Figura 2. Las cinco fuerzas competitivas

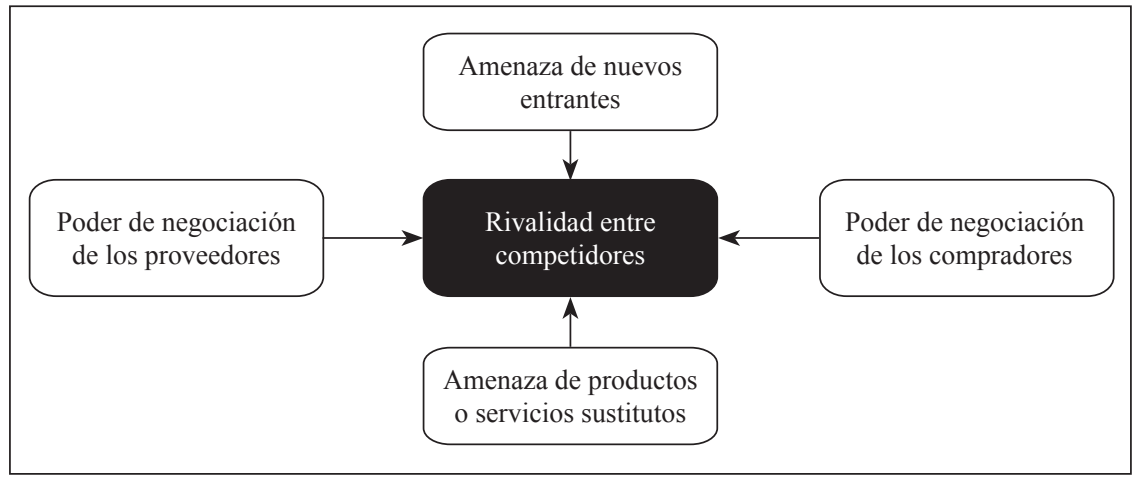

Fuente: traducida y modificada de la figura de Porter (1980, p. 4).

Con base en lo anterior, también Suárez (2008) utilizó el análisis de las cinco fuerzas competitivas para determinar qué factores externos a la Escuela de Postgrado de la Universidad Nacional de Trujillo (Perú) 
tienen afectación en sus áreas competitivas. Esto le permitió generar estrategias con el fin de obtener una ventaja competitiva sobre sus competidores. Por otra parte, Arriagada (2012) utilizó igualmente este modelo para analizar el medio externo, a través de la valoración de cada fuerza competitiva, haciendo uso de ponderaciones. Esto le permitió determinar sus fortalezas y debilidades en relación con otras universidades.

\subsection{Las universidades tecnológicas en México}

Las Universidades Tecnológicas (UT) son estructuras organizativas novedosas y con un modelo educativo diferente al tradicional, el cual es gestionado por la Coordinación General de Universidades Tecnológicas y Politécnicas (CGUTyP). Las UT son instituciones con un alto nivel académico. Esto las ha llevado a permanecer en el sector educativo durante casi veinticinco años (CGUTyP, 2006).

Un factor clave para que las UT sean efectivas es que sus directivos han tratado de utilizar al máximo sus recursos tecnológicos, humanos y financieros y de dirigir estratégicamente las instituciones a su cargo. Debido a esto, su orientación siempre ha sido la de contar con progra- mas académicos pertinentes y con un alto grado de calidad en su implementación, en el proceso de formación y en el impacto de sus egresados hacia la sociedad.

\subsection{La dirección estratégica en la universidad pública}

Dess y Lumpkin (2003, p. 3) plantean que la dirección estratégica en general es "el conjunto de análisis, decisiones y acciones que una organización lleva a cabo para crear y mantener ventajas competitivas". Thompson y Strickland (2004), por su parte, la definen como "el proceso administrativo de crear una visión estratégica, establecer los objetivos y formular una estrategia, así como implantar y ejecutar dicha estrategia"(p. 6).

De acuerdo con lo indicado por Álamo y García (2007), en su investigación sobre dirección estratégica en universidades españolas, esta tiene su origen moderno, por supuesto, en las experiencias desarrolladas en el ámbito empresarial (Andrews, 1991; Ansoff, Declerck, \& Hayes, 1976/ 1991). Estas han sido utilizadas entonces como un referente por parte de las instituciones del sector público. Este ha sido, en consecuencia, un proceso de adaptación y no de adopción. 
En este contexto, autores como Piñeiro et al. (2007), han propuesto un modelo de dirección estratégica que busca mejorar el proceso de administración en las instituciones universitarias. Lo hicieron gracias a un estudio realizado en la Escuela Internacional de Educación Física y Deporte de Cuba. Estos investigadores, como otros (Martin, 1992; Tabatoni, Davies, \& Barblan, 2002), remarcan la necesidad de que las universidades dediquen mayores esfuerzos en la aplicación de enfoques estratégicos que sean más adaptables, dinámicos y emprendedores que los que tradicionalmente han sido utilizados. Esto con el fin de poder detectar más abiertamente sus principales fuentes de ventaja competitiva y aprovechar las de forma productiva.

\section{METODOLOGÍA}

Esta investigación se desarrolló de la siguiente manera: inicialmente, se realizó una revisión documental para profundizar en los conceptos vinculados con los temas centrales del trabajo, a saber: las UT, la dirección estratégica, la cadena de valor y las cinco fuerzas definidas por Michael Porter. Con base en lo anterior, se elaboraron cuestionarios integrados por una serie de preguntas que permitieron dar continuidad a la investigación.

Para medir cada una de las variables del estudio se utilizó una escala Likert de cinco categorías, que van del extremo negativo al positivo. Estas categorías fueron enumeradas del 1 al 5, en donde 1 significa 'totalmente en desacuerdo', 2 'en desacuerdo', 3 'ni de acuerdo, ni en desacuerdo', 4 'de acuerdo' y 5 'totalmente de acuerdo'.

El concepto de dirección estratégica fue medido considerando cinco variables. Estas se encuentran determinadas en la tabla 1.

Tabla 1. Variables utilizadas para medir la dirección estratégica

\begin{tabular}{|l|l|}
\hline \multicolumn{1}{|c|}{ Variable } & \multicolumn{1}{c|}{ Identificación } \\
\hline $\begin{array}{l}\text { Misión y } \\
\text { propósitos } \\
\text { institucionales }\end{array}$ & $\begin{array}{l}\text { A través de nueve ítems específicos, diseñados con base en la definición } \\
\text { conceptual de Campbell y Nash (1992) y Rodríguez Ponce y Pedraja Rejas } \\
(2009) .\end{array}$ \\
$\begin{array}{l}\text { Esta variable se refiere al grado en que los miembros de una organización se } \\
\text { identifican con la razón de ser y existir de la misma, tanto en el momento actual } \\
\text { como de cara al futuro (Guerras y Navas, 2007). }\end{array}$ \\
\hline
\end{tabular}




\begin{tabular}{|c|c|}
\hline Variable & Identificación \\
\hline $\begin{array}{l}\text { Recursos y } \\
\text { capacidades }\end{array}$ & $\begin{array}{l}\text { A través de seis ítems específicos diseñados con base en la definición conceptual } \\
\text { de Grant (2002) y Rodríguez Ponce y Pedraja Rejas (2009). } \\
\text { Esta variable se refiere al grado en que los recursos tangibles o intangibles se } \\
\text { consideran una fuente de ventaja competitiva (Grant, 2002; Lockett \& Wright, } \\
\text { 2005; Lynch \& Baines, 2004). }\end{array}$ \\
\hline $\begin{array}{l}\text { Diseño de la } \\
\text { estrategia }\end{array}$ & $\begin{array}{l}\text { A través de cuatro ítems específicos diseñados siguiendo la definición conceptual } \\
\text { de Pedraja Rejas (2009) y Rodríguez Ponce (2005). } \\
\text { Esta variable se refiere al grado en que la estrategia permite alcanzar la misión y } \\
\text { propósitos de la institución (Bueno et al., 2002; Rodríguez Ponce, 2005; Wheelen } \\
\text { \& Hunger, 2007). }\end{array}$ \\
\hline $\begin{array}{l}\text { Implementación } \\
\text { de la estrategia }\end{array}$ & $\begin{array}{l}\text { A través de tres ítems específicos diseñados siguiendo la definición conceptual de } \\
\text { Rodríguez Ponce (2005), Rodríguez Ponce y Pedraja Rejas (2009). } \\
\text { Esta variable se refiere al grado en que las estrategias son implementadas en la } \\
\text { institución (David, 2003; Dess \& Lumpkin, 2003; Harrison, 2002; Johnson \& } \\
\text { Scholes, 1997). }\end{array}$ \\
\hline $\begin{array}{l}\text { Análisis del } \\
\text { entorno externo }\end{array}$ & $\begin{array}{l}\text { A través de cinco ítems específicos diseñados siguiendo la definición conceptual } \\
\text { de Grant (2002) y Rodríguez Ponce y Pedraja Rejas (2009). } \\
\text { Esta variable se refiere al grado en que afectan a la institución los factores económi- } \\
\text { cos, tecnológicos, sociales y gubernamentales (Grant, 2002). }\end{array}$ \\
\hline $\begin{array}{l}\text { Análisis } \\
\text { del sector } \\
\text { competitivo }\end{array}$ & $\begin{array}{l}\text { A través de seis ítems específicos, los cuales fueron diseñados para este estudio } \\
\text { con base en la definición conceptual de Porter (1980) y Rodríguez Ponce y Pedraja } \\
\text { Rejas (2009). } \\
\text { Esta variable se refiere al grado en que la institución compite en el mercado } \\
\text { (Grant, 2002; Guerras y Navas, 2007). }\end{array}$ \\
\hline
\end{tabular}

Fuente: elaboración propia.

Posteriormente, se llevó a cabo la elección de una IES pública, como objeto de estudio, considerando así a la Universidad Tecnológica Emiliano Zapata del Estado de Morelos (UTEZ), una UT representativa del sector educativo. Esta se destaca como una de las universidades con mejor calidad educativa del país. De hecho, recibió la distinción de parte de la Secretaría de Educación Pú- blica y de la Asociación Nacional de Universidades Tecnológicas en 2009, así como el Premio Estatal de Calidad y Competitividad en ese mismo año. Estos reconocimientos, entre otros aspectos, la distinguen como una entidad que ha logrado un alto desempeño, un desarrollo institucional sobresaliente y una elevada calidad educativa. De hecho, esta institución ha sido premiada por 
octavo año consecutivo por la Secretaria de Educación Pública como una universidad de excelencia académica.

Una vez realizado lo anterior, se revisaron los documentos del Plan Operativo Anual (POA). En este documento se incluye la definición de misión, visión, diagnóstico del sector, análisis del entorno externo, objetivos, estrategias y líneas de acción. Se analizó también el Programa Institucional de Desarrollo (PIDE), que contiene las bases sólidas de la planeación estratégica de la UTEZ a mediano plazo. En este se establecen las principales directrices institucionales y los procesos de la función, operación y gestión estratégica de calidad de las diversas unidades que conforman la estructura orgánica de la universidad.

Ambos documentos, junto con algunos otros complementos, los cuales ya incluyen el análisis FODA de la propia Universidad, fueron la base esencial para realizar el análisis de las fuerzas competitivas. Para la elaboración de la cadena de valor de la universidad se consideró la revisión documental de los planes operativos, así como una serie de entrevistas realizadas al personal encargado de cada actividad de la cadena. Finalmente, se realizaron también encuestas a directivos, jefes de departamento y coordinadores de área. Esta contenía preguntas semiestructuradas que se consideraban necesarias para poder profundizar en los conceptos relacionados con la problemática.

\section{RESULTADOS}

\subsection{Análisis interno utilizando la cadena de valor}

El modelo de la cadena de valor, que se muestra en la tabla 2, evidencia la interrelación existente entre las actividades primarias y de apoyo (ver tabla 3) presentes en una IES. Las actividades primarias fueron seleccionadas bajo el criterio de importancia para cumplir con la visión y la misión. Se partió desde la etapa de admisión, considerada como una actividad fundamental para obtener los objetivos, y se continuó con 1) la preparación profesional, como el proceso mediante el cual se espera desarrollar las competencias laborales y profesionales en el estudiante; 2) la vinculación con la competencia laboral, considera como el enlace entre las necesidades y requisitos del campo de trabajo, y 3) la práctica de los conocimientos. Además, se consideró la investigación y el desarrollo como un factor primario, puesto que este es visto como uno de los indicadores clave en materia de avance y autonomía de un país. 
Tabla 2. Actividades primarias de la cadena de valor de la UTEZ

\begin{tabular}{|c|c|}
\hline Actividad & Descripción \\
\hline Logística interna & $\begin{array}{l}\text { La UTEZ define la administración interna de todos los insumos necesarios para } \\
\text { crear productos de calidad alineados con la estrategia general de la institución. } \\
\text { Lo hace para que estos sean entregados a los alumnos utilizando la capacidad } \\
\text { desarrollada por los profesores y administrativos, permitiendo diseñar planes y } \\
\text { programas de estudio acordes a las necesidades del entorno. }\end{array}$ \\
\hline Operaciones & $\begin{array}{l}\text { Los productos finales de la UTEZ son las ingenierías, licencias profesionales, } \\
\text { carreras técnicas (Técnico Superior Universitario o TSU) y diplomados. Estos se } \\
\text { ofrecen para formar profesionales capacitados para innovar en la solución de los } \\
\text { problemas de la sociedad, industria o Gobierno, aportando a esto características } \\
\text { de colaboración y liderazgo. Se construye cada asignatura con sus materiales de } \\
\text { apoyo y todos los elementos descritos en la logística interna, para que pueda ser } \\
\text { entregada al alumno. Además, la UTEZ realiza todas las actividades y funciones } \\
\text { que tiene a su cargo, organizándose de acuerdo con los planes establecidos y los } \\
\text { programas de trabajo. }\end{array}$ \\
\hline $\begin{array}{l}\text { Logística } \\
\text { externa }\end{array}$ & $\begin{array}{l}\text { En esta actividad la UTEZ proporciona a los estudiantes inscritos el acceso a sus } \\
\text { cursos a través del portal de la institución. Él puede obtener en este información } \\
\text { personalizada y relevante de acuerdo con su perfil. Los portales de los estudiantes } \\
\text { son importantes para el aprendizaje en línea, ya que contribuyen a construir una } \\
\text { comunidad a distancia. En este portal, un alumno puede acceder a su cuenta de } \\
\text { registro, visualizar los cursos en los que puede certificarse, tener acceso a manua- } \\
\text { les de usuario y obtener otros servicios complementarios. Finalmente, la UTEZ } \\
\text { lleva a cabo un proceso de selección de sus mejores candidatos para que estos } \\
\text { puedan ingresar. }\end{array}$ \\
\hline $\begin{array}{l}\text { Marketing y } \\
\text { ventas }\end{array}$ & $\begin{array}{l}\text { La universidad establece un compromiso al matricular y brindar otros servicios a } \\
\text { los estudiantes inscritos en un programa en particular. El registro se considera una } \\
\text { venta, así como los materiales de aprendizaje. El factor de ingresos de la institu- } \\
\text { ción se deriva de la oferta de la enseñanza, es decir que, con base en la matrícula } \\
\text { de estudiantes inscritos se define el capital de ingresos que recibe por parte de } \\
\text { la federación. Por lo tanto, la UTEZ prepara folletos, material publicitario y un } \\
\text { calendario universitario, entre otras acciones, para promover la oferta educativa } \\
\text { y generar mayor cantidad de matrículas (que representan ingresos). La entidad } \\
\text { lleva a cabo también tareas de mantenimiento de certificaciones y evaluaciones } \\
\text { externas. Esto con el fin de publicitarlas y lograr una mayor atracción al mercado } \\
\text { estudiantil. }\end{array}$ \\
\hline $\begin{array}{l}\text { Servicios } \\
\text { posventa }\end{array}$ & $\begin{array}{l}\text { La UTEZ ofrece soporte técnico-académico en línea para los alumnos, así como } \\
\text { el servicio de educación continua. Este permite a los egresados estar al pendiente } \\
\text { de los cursos, diplomados, etc., que se ofertan en la UTEZ. Además, la UTEZ } \\
\text { mantiene una estrecha relación con las empresas que albergan a los alumnos en } \\
\text { estadía, para conocer el nivel de satisfacción de sus egresados. }\end{array}$ \\
\hline
\end{tabular}

Fuente: elaboración propia. 
Tabla 3. Actividades de soporte de la cadena de valor de la UTEZ

\begin{tabular}{|c|c|}
\hline Actividad & Descripción \\
\hline Infraestructura & $\begin{array}{l}\text { La UTEZ soporta los servicios necesarios para solventar sus necesidades gracias } \\
\text { a su infraestructura. Cuenta con cuatro edificios de docencia, una biblioteca, un } \\
\text { edificio de rectoría y dos talleres pesados, equipados con la más alta tecnología. Por } \\
\text { otro lado, existe el Centro de Vinculación y Servicios Tecnológicos (CEVISET), } \\
\text { que alberga al Centro de Desarrollo de Software (CDS) en el cual se desarrollan } \\
\text { programas informáticos con elevados estándares de calidad, bajo la norma mexica- } \\
\text { na NMX-I-059/NYCE-2005. Además, se cuenta con el Centro de Comunicación, } \\
\text { Aprendizaje y Desarrollo del Conocimiento (CECADEC). Este alberga laborato- } \\
\text { rios y centros de certificación en tecnologías como redes y voz sobre IP, entre otros. }\end{array}$ \\
\hline $\begin{array}{l}\text { Recursos } \\
\text { humanos }\end{array}$ & $\begin{array}{l}\text { Existe también el área de recursos humanos, encargada de realizar el proceso de } \\
\text { búsqueda y contratación de los profesores y otro personal requerido, consideran- } \\
\text { do el perfil adecuado. Para obtener información sobre posibles candidatos existe } \\
\text { el sitio "Bolsa de empleos UTEZ". Desde esta se invita a los exalumnos a subir y } \\
\text { mantener su currículo actualizado. Además, se cuenta con la educación continua } \\
\text { que permite el entrenamiento y desarrollo de los profesores y administrativos. }\end{array}$ \\
\hline Tecnología & $\begin{array}{l}\text { La UTEZ incluye en este rubro el manejo y desarrollo del conocimiento, mejores } \\
\text { prácticas, experiencia previa, procedimientos, investigación y selección de me- } \\
\text { jores herramientas tecnológicas y colaborativas. También hace uso de sistemas } \\
\text { informáticos especializados, como herramientas colaborativas, para gestionar ac- } \\
\text { tividades como la docencia, el deporte, la estadía y otras labores básicas. }\end{array}$ \\
\hline Compras & $\begin{array}{l}\text { La universidad se abastece por medio de proveedores internos y externos e in- } \\
\text { sumos como datos, documentos, esquemas de formación, memoria documental, } \\
\text { profesores, inteligencia corporativa, modelo pedagógico, instalaciones físicas y } \\
\text { equipamiento tecnológico, entre otros. }\end{array}$ \\
\hline
\end{tabular}

Fuente: elaboración propia.

\subsection{Análisis externo a través de las cinco fuerzas}

\section{Relacionando el modelo de las cinco} fuerzas de Porter, con los estudiantes como compradores de servicios educativos (clientes), quienes difícilmente pueden cambiar de escuela dado el alto costo económico en transporte (si tienen que trasladarse), nuevo material, colegiaturas (si es privada), el cambio de círculo social y la revalidación en su retícula académica, entre otros. Esta fuerza de negociación es considerada como BAJA. Su poder reside en el hecho de que el estudiante elije a qué tipo de IES se inscribe.

Por otra parte, la UTEZ, como institución pública, no tiene proveedores cuyo poder afecte al servicio 
educativo. Esto dado que el carácter de este producto es intangible y no depende de materias primas que condicionan su valor final. La Secretaría de Educación Pública (SEP) es quien gestiona el desarrollo del sector educativo sostenido con fondos públicos. Esta controla además todo el sector educativo, ya sea público o privado. De hecho, la SEP supervisa y da el visto bueno para que los centros educativos puedan ejercer su función. Su carácter no es competitivo, sino cooperativo, pues su interés es el bien del usuario final.

La universidad tiene como proveedores el servicio de limpieza, la cafetería, el mantenimiento de instalaciones eléctricas y reparaciones en general, pues estos son servicios externos por los que la UTEZ paga de su presupuesto anual. Estos proveedores tienen un poder muy BAJO respecto a la universidad. Aun siendo importante el servicio que prestan, la capacidad de ser sustituidos por otros iguales es muy ALTA.

En la actualidad, el estado de Morelos vive un incremento de la demanda educativa propiciado por la reactivación de la economía y una mejor marcha del sector empresarial. El incremento de la demanda supone nuevas oportunidades para aquellos competidores que quieren entrar por primera vez al sector educativo. Las barreras de entrada para los centros educativos privados es ALTA. Esto debido a los costos de inversión en el inmueble, el profesorado y las cuotas exigidas para su instalación. Por esto, la incursión de algún nuevo centro educativo privado en Morelos no alteraría significativamente la intensidad competitiva en el sector público, pues la forma de competir en este contexto es diferente.

La principal barrera de entrada para todos los centros educativos públicos es la política gubernamental, que depende de la Secretaría de Educación del Estado, de sus criterios y presupuestos. Actualmente, se identifica como producto sustituto el método autoinstruccional. Sin embargo, en cualquier momento podrían surgir otros, dada la evolución de las tecnologías de la información y la comunicación (TIC) y los diferentes enfoques que las nuevas generaciones puedan dar al Internet. Por esta razón, esta amenaza se considera MEDIA.

\subsection{Resultados obtenidos de la encuesta sobre dirección estratégica}

A continuación, se presentan los resultados más sobresalientes de las encuestas aplicadas al personal responsable de la planeación estratégica de la UTEZ. 
Figura 3. Histograma de análisis del entorno general

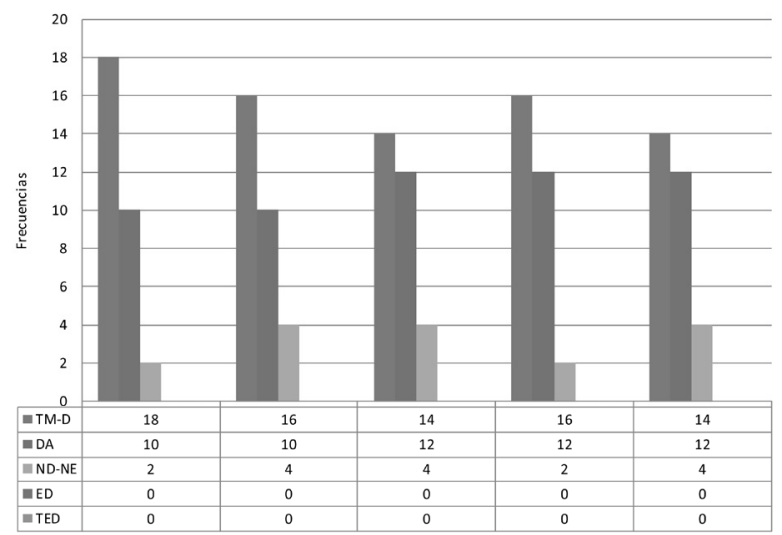

Fuente: elaboración propia.

En la figura 3 se puede apreciar que de los 150 reactivos recibidos para este rubro, se encontró que 78 corresponden a que los actores están totalmente de acuerdo (TM-D) en que el análisis del entorno general está plenamente orientado a conocer los factores que rodean a la univer- sidad, tanto políticos, como sociales, económicos, culturales y tecnológicos. Estos representan el 52\% del total de reactivos. Cabe señalar que tan solo 16 estuvieron presentes en el criterio de ni de acuerdo, ni en desacuerdo (ND-NE), lo que representa solo el $10,66 \%$ del total.

Figura 4. Histograma del análisis del entorno competitivo

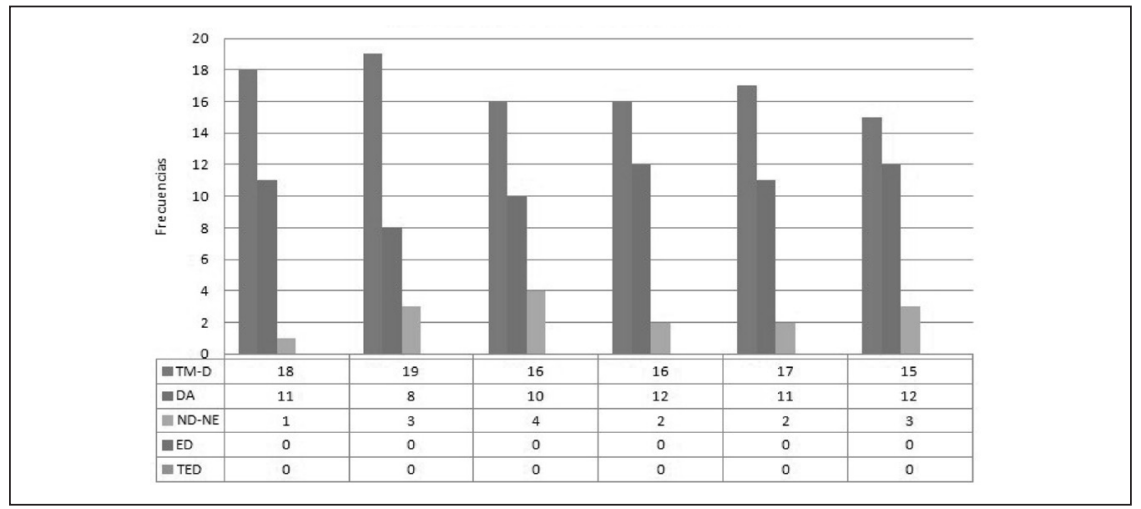

Fuente: elaboración propia. 
En la figura 4 se puede apreciar que de los 180 reactivos recibidos para este rubro, se encontró que 101 corresponden a que los actores están totalmente de acuerdo (TM-D) en que el análisis del entorno competitivo está enfocado en conocer los factores que influyen en la universidad, como la competencia, la rivalidad entre universidades del mismo tipo, la sustitución de sus servicios y el poder de negociación de los compradores. Cabe señalar que tan solo 15 reactivos estuvieron presentes en el criterio de ni de acuerdo, ni en desacuerdo (ND-NE), lo que representa solo el $8,33 \%$ del total.

Figura 5. Histograma del análisis de recursos y capacidades

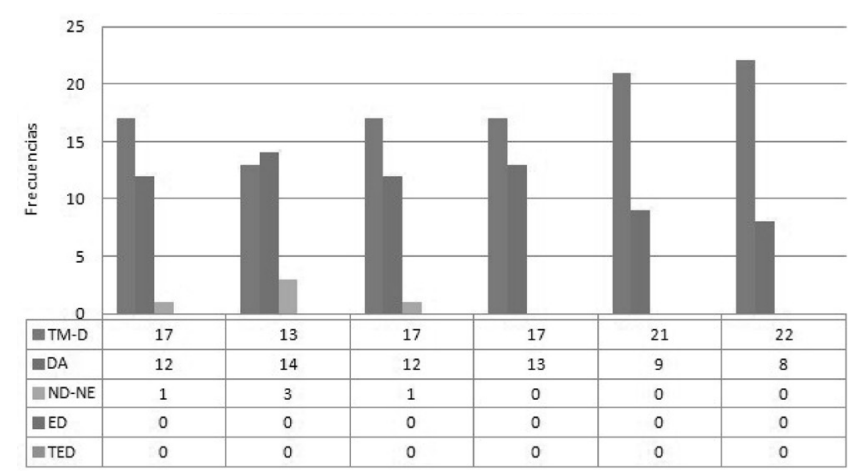

Fuente: elaboración propia.

Por último, en la figura 5 , se puede apreciar que de los 180 reactivos recibidos para este rubro, se encontró que 107 corresponden a que los actores están totalmente de acuerdo (TM-D) en que el análisis de los recursos y capacidades está orientado a conocer los factores que influyen para mantener el talento humano en la universidad, con el fin de obtener ventaja competitiva a través de ellos. Estos reactivos representan el $59,44 \%$ del total. Cabe señalar que tan solo 5 reactivos estuvieron pre- sentes en el criterio de ni de acuerdo, ni en desacuerdo (ND-NE), lo que representa solo el $2,77 \%$ del total. Con base en las gráficas anteriores, se puede deducir que la dirección estratégica en la UTEZ, en efecto, está siendo adoptada como una forma de trabajo cotidiana.

\section{CONCLUSIONES}

En esta investigación, se pudo constatar que la UTEZ tiene definidas las actividades primarias y de soporte de 
la cadena de valor. Además, fue posible identificar los factores externos que pueden afectar el desarrollo de las estrategias de la institución. Con la información analizada se detectaron áreas de oportunidad que podrían ser desarrolladas para mejorar la tarea de formación con servicios complementarios. Se descubrieron también las principales debilidades y amenazas, las cuales se pueden atacar con las estrategias resultantes que fueron propuestas a partir del análisis FODA.

La UTEZ debería pronosticar la demanda esperada para disponer de los elementos tecnológicos, recursos e insumos necesarios con el fin de optimizar cada punto de su cadena de valor y asegurar un servicio que proporcione calidad y satisfacción a los alumnos a lo largo de su interacción con la institución.

Para que la institución mantenga su ventaja competitiva es necesario que fortalezca más sus relaciones con universidades extranjeras, utilizar la subcontratación en actividades secundarias, ampliar su mercado y mejorar la satisfacción de sus clientes mediante innovaciones continuas. La información recabada de las encuestas ayudó a consolidar el análisis FODA, así como a determinar la estructura de la cadena de valor y rea- lizar el análisis de las cinco fuerzas competitivas. Las encuestas son el reflejo de la apreciación que tiene el personal sobre la forma distinta en cómo se está dirigiendo a la universidad, es decir, a través del uso de técnicas y herramientas propias de la dirección estratégica.

Se puede concluir este trabajo afirmando que la UTEZ se está profesionalizando cada vez más, haciendo uso de modernas técnicas de administración y dirección de organizaciones. Estas le permiten conocerse mejor y comprender de una manera más amplia su entorno y a las organizaciones tanto públicas como privadas con las que compite.

Con la investigación que aquí se presenta, se pretende servir de guía a instituciones que aún no hayan emprendido su proceso de dirección estratégica y a aquellas que en su primera aproximación se acercaron tímidamente a esta técnica de dirección, pero no profundizaron en su utilización ni avanzaron significativamente en su aplicación. Finalmente, los resultados de la utilización de herramientas como la dirección estratégica no pueden observarse a corto plazo, esto a pesar de que la satisfacción con su aplicación pueda llegar a valorarse tan pronto como el primer año después de su implementación. 


\section{REFERENCIAS}

Álamo, F., \& García, M. (2007). El proceso estratégico en el sector público: Análisis en el contexto de las universidades españolas. Investigaciones Europeas de Dirección y Economía de la Empresa, 13(2), 113-129.

Andrews, K. R. (1991). El concepto de estrategia corporativa. En H. Mintzberg \& J.B.Quinn(Eds.), El proceso estratégico: conceptos, contextos y casos (2a ed., pp. 5160). México: Prentice Hall.

Ansoff,H. I., Declerck, R. P., \& Hayes, R. L. (1976/1991). De la planeación estratégica a la administración estratégica. En H. I. Ansoff, R. P. Declerck \& R. L. Hayes (Eds.), El planteamiento estratégico: nueva tendencia de la administración (pp. 49-91). México: Trillas.

Arriagada, M. (2012). Plan de negocios para la fundación Daniela Aspillaga. Magíster en Gestión y Dirección de Empresas, tesis de maestría inédita, Universidad de Chile, Santiago de Chile.

Bueno, E., Morcillo, P., Rodríguez, J., Luque, M., Cervera, M., \& Rodríguez, O. (2002). Indicadores de capital intelectual aplicados a la actividad investigadora y de gestión del conocimiento en las universidades y centros públicos de investigación de Madrid. Madrid: Dirección General de Investiga- ción, Consejería de Educación, Comunidad de Madrid.

Bradshaw, P., \& Fredette, C. (2009). Academic governance of universities reflections of a senate chair on moving from theory to practice and back. Journal of Management Inquiry, 18(2), 123-133.

Campbell, A., \& Nash, L. (1992). A sense of mission: Defining direction for the large corporation. Reading: Addison-Wesley.

Castaño,N.(2004).Universidad-Empresa la oportunidad que tiene Colombia para innovar en tecnologías de la información. Ingeniería de Sistemas. Bogotá: Pontificia Universidad Javeriana.

Capriotti, P. (1999). Planeación estratégica de la imagen corporativa. Malaga: Instituto de Investigación en Relaciones Públicas.

Coordinación General de UniversidadesTecnológicasyPolitécnicas (CGUTyP)(2006). 15 años, 1991 2006. Universidades Tecnológicas. Impulsando el desarrollo de México [Libro conmemorativo]. México: CGUT-SEP.

David, F. (2003). Conceptos de administración estratégica. México: Pearson Education.

Dess, G., \& Lumpkin, G. (2003). Dirección estratégica: creando ventajas competitivas. Madrid: McGraw Hill.

Grant, R.(2002). Contemporarystrategy analysis: Concepts, techni- 
ques, applications. Cambridge: Blackwell Publishers.

Guerras, M., \& Navas, J. (2007). La dirección estratégica: teoría y aplicaciones. Madrid: ThomsonCivitas.

Gumbau, J.(2006). Hacia la universidad orientada a los servicios: una perspectiva sistémica de cambio permanente por la innovación tecnológica. Revista de Universidad y Sociedad del Conocimiento, 3(1), 21-43.

Harrison, S. (2002). Fundamentos de la dirección estratégica. Madrid: Internacional Thomson Editores Johnson, G., \& Scholes, K. (1997). Direcciónestratégica: análisis de la estrategia de las organizaciones. Madrid: Prentice Hall.

Lockett, A., \& Wright, M. (2005). Resources, capabilities, risk capital and the creation of university spinout companies. Research policy, 34(7), 1043-1057.

Lynch, R., \& Baines, P. (2004). Strategy development in UK higher education: Towards resource-basedcompetitiveadvantages.Journal of Higher Education Policy and Management, 2, 172-187.

Martin, M. (1992). Strategic management in Western European universities. París: International Institute for Educational Planning. McCormick, D. (2005). El futuro de los clusters y las cadenas productivas. Semestre económico, 8(15), 87-102.

Olssen,M.,\&Peters, M.(2005).Neoliberalism, higher education and the knowledge economy: From the free market to knowledge capitalism. Journal of Education Policy, 20(3), 313-345.

Piñeiro, N., Calderón, P, Celestrín, R., García, G.,Lamas,L., Fornaris,L., \& Borroto, O. (2007). Dirección estratégica: Experiencia de la escuela internacional de educación física y deporte. La Habana: Universidad de la Habana.

Porter, M. (1980). Competitive Strategy. New York: Free Press.

Porter, M. (1985). Competitive Advantage. New York: Free Press.

Porter, M. E. (2008). The five competitive forces that shape strategy. Harvard Business Review, 86(1), 78-93.

Rodríguez Ponce, E. (2005). La toma de decisiones estratégicas en las instituciones universitarias. Un estudio empírico en Chile y España. Santiago de Chile: Editorial Andros.

Rodríguez Ponce, E., \& Pedraja Rejas, L. (2007). Efectos e implicaciones de las decisiones estratégicas en las instituciones universitarias. RevistaInterciencia, 32, 593-600. Rodríguez Ponce, E., \& Pedraja Rejas, L. (2009). Dirección estratégica en universidades: un estudio 
empírico en instituciones de Iberoamérica. Revista Interciencia, 34(6), 413-418.

Santos, J. (2005). Análisis y planeamiento de la cadena de valor general de la Universidad de la Sabana. Ingeniería Industrial, trabajo de grado inédito, Universidad de la Sabana, Chía, Colombia. Suárez,C.(2008).Implementaciónde un cuadro de mando integral y su influencia en la productividad de la escuela de postgrado de la universidad nacional de Trujillo. Magíster en Ingeniería, tesis de maestría inédita, Universidad Nacional de Trujillo. Trujillo, Perú. Tabatoni, P., Davies, J., \& Barblan, A. (2002). Strategicmanagementand universities' institutional development. Genève: European University Association.

Thompson,A.yStrickland,J.(2004). Strategic management: Concepts and cases. Nueva York: McGrawHill.

Wheelen,L.\&Hunger,D.(2007).Administración estratégica y políticadenegocios: conceptosycasos. México: Pearson Education. 